\title{
Prognostic factors in patients with pancreatic cancer
}

\author{
DE-XIANG ZHANG ${ }^{1}$, YUE-DI DAI ${ }^{2}$, SU-XU YUAN $^{3}$ and LITAO ${ }^{2}$ \\ ${ }^{1}$ Department of General Surgery, Jinshan Hospital Affiliated to Fudan University, Shanghai 200540; \\ ${ }^{2}$ Department of Medical Oncology, Cancer Hospital of Fudan University, Minhang Branch, Shanghai 200240; \\ ${ }^{3}$ Department of Medical Oncology, the First Affiliated Hospital of Soochow University, Suzhou 215006, P.R. China
}

Received August 22, 2011; Accepted November 18, 2011

DOI: $10.3892 / \mathrm{etm} .2011 .412$

\begin{abstract}
The identification of prognostic factors for pancreatic cancer patients could provide insightful information for their management in the clinic. A total of 302 pancreatic cancer patients were enrolled in this study. The clinicopathological characteristics, treatment selection and laboratory test data were retrospectively retrieved from the medical records and follow-up data were obtained via telephone interview. Cox survival analysis was used to assess the potential prognostic factors, and survival curves were obtained by Kaplan-Meier analyses. The mortality rate of the patients was $83.4 \%(252 / 302)$ and the median survival of these patients was 6.1 months, with 1-, 2- and 3-year survival rates of 30.1 (91/302), 10.6 (32/302) and $2.6 \%$ (8/302), respectively. The most influential factors for the survival of these patients were the site of primary cancer, tumor stage, treatment selection, serum levels of glutamic-pyruvic transaminase, albumin, lactate dehydrogenase and hemoglobin, and white blood cell counts $(\mathrm{P}<0.05)$. The median survival of patients who did not receive any treatment or just received supportive treatment was 1.3 months, while the median overall survival of patients who underwent surgery, chemotherapy, biliary drainage therapy, arterial interventional chemotherapy and comprehensive treatment was 11.0, 7.3, 3.5, 9.0 and 11.0 months, respectively $(\mathrm{P}<0.05)$. Furthermore, single-drug chemotherapy was not statistically associated with patient survival in those who received the multi-drug regimen $(\mathrm{P}>0.05)$. However, the mortality risk of patients who received platinum chemotherapy was decreased [hazard ratio $(\mathrm{HR})=0.56,95 \% \mathrm{CI} 0.35-0.88, \mathrm{P}=0.011]$ compared to the patients who did not receive this treatment $(\mathrm{P}<0.05)$. Tumor stage, treatment selection, serum albumin levels, urea nitrogen, CA19-9, white blood cell and platelet counts were independent prognostic factors for the prediction of survival in pancreatic cancer. Future studies are required in order to verify these data.
\end{abstract}

Correspondence to: Dr Yue-Di Dai, Department of Medical Oncology, Cancer Hospital of Fudan University, Minhang Branch, Shanghai 200240, P.R. China

E-mail: dai.yue.di@hotmail.com

Key words: pancreatic cancer, clinicopathological parameters, treatment selection, prognostic factor
Chemotherapy with platinum regimens could improve overall survival in patients with pancreatic cancer.

\section{Introduction}

Pancreatic cancer is a lethal disease due to late-stage diagnosis and a lack of effective treatment strategies. The prognosis of patients with pancreatic cancer is very poor, with a median survival of only 3-5 months and 1-year survival of less than $10 \%(1,2)$. As with the majority of solid tumors, surgery is the best choice for treating pancreatic cancer; however, up to $80 \%$ of pancreatic cancer patients are diagnosed at an advanced stage, which makes surgery difficult and ineffective. To date, curative resection of pancreatic cancer occurs in only 10-15\% of patients (3). Moreover, chemotherapy is only palliative to improve quality of life and gain a modest survival benefit, and is used when patients are not suitable for surgery. Accumulating evidence suggests that successful surgical resection is one of the most crucial factors in the effective treatment of pancreatic cancer (4), while chemotherapy and radiotherapy are most effective in the treatment of locally unresectable and recurrent pancreatic cancer (5). Therefore, novel strategies that aid in the early detection, disease prevention, effective treatment and prognosis prediction of this deadly disease are required.

Pancreatic cancer development is likely associated with the silencing of tumor suppressor genes and the activation of oncogenes, although the underlying molecular mechanisms remain to be defined. The altered expression of these genes may not only trigger the development of pancreatic cancer but also promote tumor progression, leading to a poor prognosis. Indeed, there have been a number of studies published that associate the development and prognosis of pancreatic cancer with a variety of factors (6). For example, a recent Japanese study calculated the Onodera's prognostic nutrition index (PNI), based on serum albumin and three other constitutive indices, to be an independent prognostic factor useful in the prediction of survival of pancreatic cancer patients (7). In addition, other studies have focused on utilizing the characteristics of the tumor phenotype, such as the number of metastatic lymph nodes (8), tumor invasion or adhesion to peripancreatic blood vessels and positive margins following pancreatoduodenectomy $(9,10)$ as prognostic factors for pancreatic cancer. However, to date, there are no studies found to associate the clinicopathological characteristics with pancreatic cancer prognosis. Furthermore, there have been no reports of the selection of pancreatic cancer 
treatment with varying prognoses. Therefore, this study retrospectively evaluated the clinicopathological features, treatment selection and laboratory test data for pancreatic cancer to guide its future management and to predict its prognosis.

\section{Materials and methods}

Patient population. In this study, we retrospectively reviewed data from pancreatic cancer patients from the Cancer Hospital Affiliated to Fudan University, Minhang Branch, The First Affiliated Hospital of Suzhou University and the Jinshan Hospital Affiliated to Fudan University, between January 2003 and December 2009. A total of 415 patients were identified and cytohistologically diagnosed to have pancreatic cancer; 302 of them received a follow-up to ensure survival via a phone interview. The overall survival was defined as the period from the cytohistological diagnosis to the date of mortality or the most recent follow-up. The institutional review boards at our institutions approved this study and all patients or their legal guardians signed an informed consent form.

Clinical data. The data of clinicopathological characteristics were retrieved from the patients' medical records and follow-up data were also obtained via phone interview. Of these 302 pancreatic cancer patients, 189 (63\%) were male and $113(37 \%)$ were female, and the median age was 63 years with a range of 28-89 years. A total of 140 patients had a pathological diagnosis determined from surgical specimens, 85 patients were diagnosed through tissue biopsy of metastatic or primary lesions, 32 cases via tissue biopsy from liver metastasis puncture, 18 patients via cytological examination of pleural effusion or ascites and 27 patients failed to provide definitive pathological diagnosis. Moreover, 251 patients had pancreatic adenocarcinoma, 5 had other types of cancer and 46 did not provide definitive cytological results to diagnose the type of cancer. In total, 189 cancers were located at the head or neck of the pancreas, 110 cases at the body and tail of the pancreas and 3 cases had spread to the entire pancreas. In addition, 8 cases were diagnosed as Stage I, 34 cases as Stage II, 94 as Stage IV and 166 cases as Stage V, according to the 2002 guidelines of the American Joint Committee on Cancer (AJCC). A total of 67 patients did not receive any treatment or only supportive treatment following diagnosis, 140 patients underwent surgical treatment (47 patients underwent radical surgery, 74 underwent palliative surgery and 19 did not have definitive surgical modalities), 115 patients underwent chemotherapy, 17 underwent biliary drainage, 57 underwent arterial interventional chemotherapy, 3 underwent local radiotherapy and 86 underwent comprehensive treatment.

Statistical analyses. Survival curves were constructed by the Kaplan-Meier method. Cox survival univariate and multivariate analyses were performed to identify potential prognostic factors based on the determination of the most significant variables that may contribute to survival. Two-sided P-values of $<0.05$ were considered to indicate a statistically significant difference. The association between different clinicopathological characteristics or their significance to survival was analyzed using the Chi-square test. All statistical analyses were performed using the SPSS statistical software program package (SPSS version 13.0 for windows; SPSS Inc., Chicago, IL, USA).

\section{Results}

Identification of prognostic factors for pancreatic cancer patients. Among these 302 pancreatic cancer patients, 252 had succumbed to the disease since their last follow-up and the mortality rate was $83.4 \%$. The median survival period for these 302 patients was 6.1 months (Fig. 1), with 1-, 2- and 3 -year survival rates of 30.1 (91/302), 10.6 (32/302) and 2.6\% (8/302), respectively.

The overall survival data by Cox univariate regression analysis are presented in Table I. The most influential factors predicting the survival of these patients were the site of primary cancer, tumor stage, treatment selection, the levels of serum glutamic-pyruvic transaminase (GPT), serum albumin, serum lactate dehydrogenase (LDH) and hemoglobin, and white blood cell (WBC) counts $(\mathrm{P}<0.05)$. In particular, the mortality risk was increased for pancreatic cancer masses located at the body and tail of the pancreas compared to the tumors located at the head and neck of the pancreas [hazard ratio $(\mathrm{HR})=1.37$, 95\% CI 1.08-1.75, P=0.01; Fig. 1). The mortality risk was also increased in pancreatic cancer that was in advanced stages compared to early stages (HR=1.64, $95 \%$ CI $1.37-1.96, \mathrm{P}=0.000$; Fig. 1). However, the mortality risk of patients who underwent surgery, chemotherapy, biliary drainage, arterial interventional chemotherapy or comprehensive treatment was reduced compared to the patients who did not receive any treatment or who just received supportive treatment (HR $=0.84,95 \%$ CI 0.77-0.91, $\mathrm{P}=0.000$; Fig. 2). The mortality risk of patients with high GPT levels was also reduced compared to the patients with normal levels (HR=0.77, 95\% CI 0.59-0.99, $\mathrm{P}=0.042$; Fig. 3). The mortality risk of the patients with high or normal albumin levels was reduced compared to the patients with low levels (HR=0.60, 95\% CI 0.46-0.79, P=0.000; Fig. 4). In addition, the mortality risk of the patients with high serum LDH levels was increased compared to the patients with normal levels (HR=1.50, 95\% CI 1.13-1.99, $\mathrm{P}=0.005$; Fig. 4). The mortality risk of patients with normal hemoglobin levels was reduced compared to the patients with low levels $(\mathrm{HR}=0.73$, 95\% CI 0.55-0.96, P=0.023; Fig. 5). The mortality risk of patients with high WBC counts was increased compared to patients with normal or low counts (HR=1.63, $95 \%$ CI 1.272.09, $\mathrm{P}=0.000$; Fig. 5). Other factors, such as gender, age, the levels of serum total bilirubin (TB), serum direct bilirubin (DB), serum glutamic-oxalacetic transaminase (GOT) (Fig. 3), blood urea nitrogen (BUN), serum creatinine (Fig. 6), blood glucose (Fig. 4) and platelet counts, had no association with patient survival $(\mathrm{P}>0.05)$.

Analysis of tumor markers indicated that the patients with high serum CEA levels had a median survival of 2.0 vs. 5.0 months in patients with normal levels, which was statistically significant (HR=1.43, 95\% CI 1.04-1.98, $\mathrm{P}=0.030$; Fig. 7). Patients with high serum CA125 levels had a median survival of 3.1 vs. 10.1 months in patients with normal levels $(\mathrm{HR}=2.06,95 \%$ CI 1.13-3.76, $\mathrm{P}=0.018$; Fig. 7). Patients with high serum CA19-9 levels had a median survival of 3.8 months vs. 5.0 months in patients with normal levels $(\mathrm{HR}=1.41$, 95\% CI 0.99-2.02, P=0.060; Fig. 7). However, the serum levels 
Table I. Cox univariate regression analysis of 302 pancreatic cancer patients.

\begin{tabular}{|c|c|c|c|c|}
\hline Parameter & No. & Median survival (months; 95\% CI) & P-value & Hazard ratio $(95 \% \mathrm{CI})$ \\
\hline \multicolumn{5}{|l|}{ Gender } \\
\hline Male & 189 & $6.0(5.0-7.4)$ & & \\
\hline Female & 113 & $5.1(3.7-8.0)$ & 0.342 & $1.13(0.88-1.47)$ \\
\hline \multicolumn{5}{|l|}{ Age (years) } \\
\hline$\leq 60$ & 119 & $7.1(5.3-10.4)$ & & \\
\hline$>60$ & 183 & $5.0(4.0-7.0)$ & 0.331 & $1.01(0.99-1.02)$ \\
\hline \multicolumn{5}{|l|}{ Site of primary cancer } \\
\hline Head and neck & 189 & $7.6(6.0-10.6)$ & & \\
\hline Body and tail & 110 & $4.0(3.3-5.0)$ & & \\
\hline Whole pancreas & 3 & $1.7(1.1-3.0)$ & 0.010 & $1.37(1.08-1.75)$ \\
\hline \multicolumn{5}{|l|}{ Stage } \\
\hline I & 8 & $20.0(12-34.3)$ & & \\
\hline II & 34 & $10.6(6.0-20.0)$ & & \\
\hline III & 94 & $10.4(7.0-14.0)$ & & \\
\hline IV & 166 & $4.0(3.0-5.0)$ & 0.000 & $1.64(1.37-1.96)$ \\
\hline \multicolumn{5}{|l|}{ Treatment selection } \\
\hline Supportive treatment & 67 & $1.3(1.0-3.0)$ & & \\
\hline Surgery, chemo- or interventional therapy & 235 & $7.4(6.0-9.3)$ & 0.000 & $0.84(0.77-0.91)$ \\
\hline \multicolumn{5}{|l|}{ Total bilirubin } \\
\hline Normal & 144 & $5.3(4.3-7.3)$ & & \\
\hline High & 158 & $6.0(5.0-9.0)$ & 0.362 & $0.89(0.69-1.15)$ \\
\hline \multicolumn{5}{|l|}{ Direct bilirubin } \\
\hline Normal & 58 & $7.0(4.0-11.0)$ & & \\
\hline High & 244 & $5.7(5.0-7.0)$ & 0.386 & $1.16(0.83-1.61)$ \\
\hline \multicolumn{5}{|l|}{ Glutamic-pyruvic transaminase } \\
\hline Normal & 164 & $5.0(4.0-7.0)$ & & \\
\hline High & 136 & $7.0(5.1-11.0)$ & 0.042 & $0.77(0.59-0.99)$ \\
\hline \multicolumn{5}{|l|}{ Glutamic-oxalacetic transaminase } \\
\hline Normal & 170 & $5.7(5.0-7.3)$ & & \\
\hline High & 130 & $6.0(4.2-11.0)$ & 0.173 & $0.84(0.65-1.08)$ \\
\hline \multicolumn{5}{|l|}{ Albumin } \\
\hline Low & 82 & $1.8(1.23-5.0)$ & & \\
\hline Normal & 210 & $7.0(5.4-9.0)$ & & \\
\hline High & 7 & $14.2(3.0-27.1)$ & 0.000 & $0.60(0.46-0.79)$ \\
\hline \multicolumn{5}{|l|}{ Blood urea nitrogen } \\
\hline Normal & 270 & $6.0(5.0-7.1)$ & & \\
\hline High & 29 & $1.7(0.9-13.1)$ & 0.854 & $0.96(0.61-1.50)$ \\
\hline \multicolumn{5}{|l|}{ Serum creatinine } \\
\hline Normal & 263 & $6.6(5.0-8.0)$ & & \\
\hline High & 36 & $4.3(1.2-6.0)$ & 0.443 & $1.17(0.78-1.75)$ \\
\hline \multicolumn{5}{|l|}{ Lactate dehydrogenase } \\
\hline Normal & 200 & $7.0(5.8-9.0)$ & & \\
\hline High & 83 & $4.3(1.7-4.4)$ & 0.005 & $1.50(1.13-1.99)$ \\
\hline \multicolumn{5}{|l|}{ Blood glucose } \\
\hline Low & 16 & $3.0(1.0-7.0)$ & & \\
\hline Normal & 185 & $7.0(5.0-9.3)$ & & \\
\hline High & 96 & $5.0(3.2-7.3)$ & 0.965 & $0.99(0.77-1.28)$ \\
\hline \multicolumn{5}{|l|}{ Hemoglobin } \\
\hline Low & 89 & $3.7(2.2-5.7)$ & & \\
\hline Normal & 212 & $7.0(5.4-9.0)$ & 0.023 & $0.73(0.55-0.96)$ \\
\hline
\end{tabular}


Table I. Continued.

\begin{tabular}{|c|c|c|c|c|}
\hline Parameter & No. & Median survival (months; 95\% CI) & P-value & Hazard ratio $(95 \% \mathrm{CI})$ \\
\hline \multicolumn{5}{|c|}{ White blood cells } \\
\hline Low & 33 & $10.6(5.3-18.4)$ & & \\
\hline Normal & 212 & $7.0(5.1-8.0)$ & & \\
\hline High & 56 & $1.7(1.0-3.3)$ & 0.000 & $1.63(1.27-2.09)$ \\
\hline \multicolumn{5}{|l|}{ Platelets } \\
\hline Low & 52 & $3.5(2.0-7.0)$ & & \\
\hline Normal & 220 & $7.0(5.1-8.0)$ & & \\
\hline High & 29 & $5.4(2.2-13.0)$ & 0.332 & $0.88(0.68-1.14)$ \\
\hline \multicolumn{5}{|l|}{ CEA } \\
\hline Normal & 130 & $5.0(4.0-6.0)$ & & \\
\hline High & 67 & $2.0(1.3-3.0)$ & 0.030 & $1.43(1.04-1.98)$ \\
\hline \multicolumn{5}{|l|}{ CA19-9 } \\
\hline Normal & 52 & $5.0(3.0-9.1)$ & & \\
\hline High & 153 & $3.8(2.5-5.0)$ & 0.060 & $1.41(0.99-2.02)$ \\
\hline \multicolumn{5}{|l|}{ CA125 } \\
\hline Normal & 22 & $10.1(6.0-18.0)$ & & \\
\hline High & 26 & $3.1(1.4-5.0)$ & 0.018 & $2.06(1.13-3.76)$ \\
\hline \multicolumn{5}{|l|}{ CA15-3 } \\
\hline Normal & 47 & $2.4(1.6-5.0)$ & & \\
\hline High & 26 & $3.2(1.2-7.8)$ & 0.564 & $0.86(0.50-1.46)$ \\
\hline \multicolumn{5}{|l|}{ CA72-4 } \\
\hline Normal & 40 & $1.4(1.0-2.5)$ & & \\
\hline High & 13 & $1.6(0.8-2.0)$ & 0.429 & $1.33(0.66-2.69)$ \\
\hline \multicolumn{5}{|l|}{ CA50 } \\
\hline Normal & 31 & $1.7(0.8-4.3)$ & & \\
\hline High & 29 & $1.3(0.8-1.6)$ & 0.258 & $1.40(0.78-2.49)$ \\
\hline \multicolumn{5}{|l|}{ CA242 } \\
\hline Normal & 15 & $1.2(0.6-2.4)$ & & \\
\hline High & 27 & $1.6(0.8-3.7)$ & 0.312 & $0.70(0.35-1.40)$ \\
\hline
\end{tabular}

of CA15-3, CA72-4, CA50 and CA242 were not associated with survival $(\mathrm{P}>0.05)$.

Cox multivariate regression analysis data of the overall survival are presented in Table II. The most influential factors were tumor stage, treatment selection, levels of serum albumin and BUN (Fig. 6), WBC and platelet counts, and serum CA19-9 levels $(\mathrm{P}<0.05)$. In brief, the mortality risk of patients with advanced stage disease was increased by $46 \%$ (HR $=1.46$, 95\% CI 1.19-1.78, $\mathrm{P}=0.000$ ). The mortality risk of patients who underwent surgery, chemotherapy, interventional therapy, biliary drainage or comprehensive treatment was reduced by $49 \%$ compared to patients who did not undergo treatment or only received supportive treatment $(\mathrm{HR}=0.51,95 \% \mathrm{CI}$ $0.36-0.73, \mathrm{P}=0.000$ ). The mortality risk of patients with high levels of serum albumin, BUN, platelets and CA19-9 was reduced by $38,57,27$ and $53 \%$, respectively, and the mortality risk of patients with high WBC counts was increased by $49 \%$ $(\mathrm{P}<0.05)$. However, other factors, such as gender, age, site of primary cancer, the levels of TB, DB, GPT, GOT, serum creatinine, serum LDH, blood glucose and hemoglobin, were not associated with mortality risk $(\mathrm{P}>0.05)$.
Effects of treatment selection on overall survival of pancreatic cancer patients. The survival of the patients who underwent supportive treatment or no treatment was used as the control and the various treatment selections vs. the survival of the patients are presented in Table III and Fig. 2. In particular, the median survival of patients who did not receive any treatment or just received supportive treatment was 1.3 months, while the median overall survival of patients who underwent surgery, chemotherapy, biliary drainage therapy, arterial interventional chemotherapy and comprehensive treatment was 11.0, 7.3, 3.5, 9.0 and 11.0 months, respectively. The mortality risk for these treatments decreased by $61 \%(95 \% \mathrm{CI} 0.28-0.55, \mathrm{P}=0.000)$, $52 \%$ (95\% CI $0.35-0.68, \mathrm{P}=0.000$ ), $52 \%$ (95\% CI $0.24-0.94$, $\mathrm{P}=0.031), 57 \%(95 \% \mathrm{CI} 0.29-0.63, \mathrm{P}=0.000)$ and $72 \%(95 \% \mathrm{CI}$ $0.26-0.54, \mathrm{P}=0.000$ ), respectively.

A comparison of the overall survival between radical surgery and palliative surgery treatment is presented in Table IV and Fig. 2. In brief, the median survival of patients receiving radical surgery was 17.6 months vs. 8.3 months in patients receiving palliative surgery; these data did not reach statistical significance (HR=1.50; 95\% CI 0.98-2.29, $\mathrm{P}=0.065)$. 

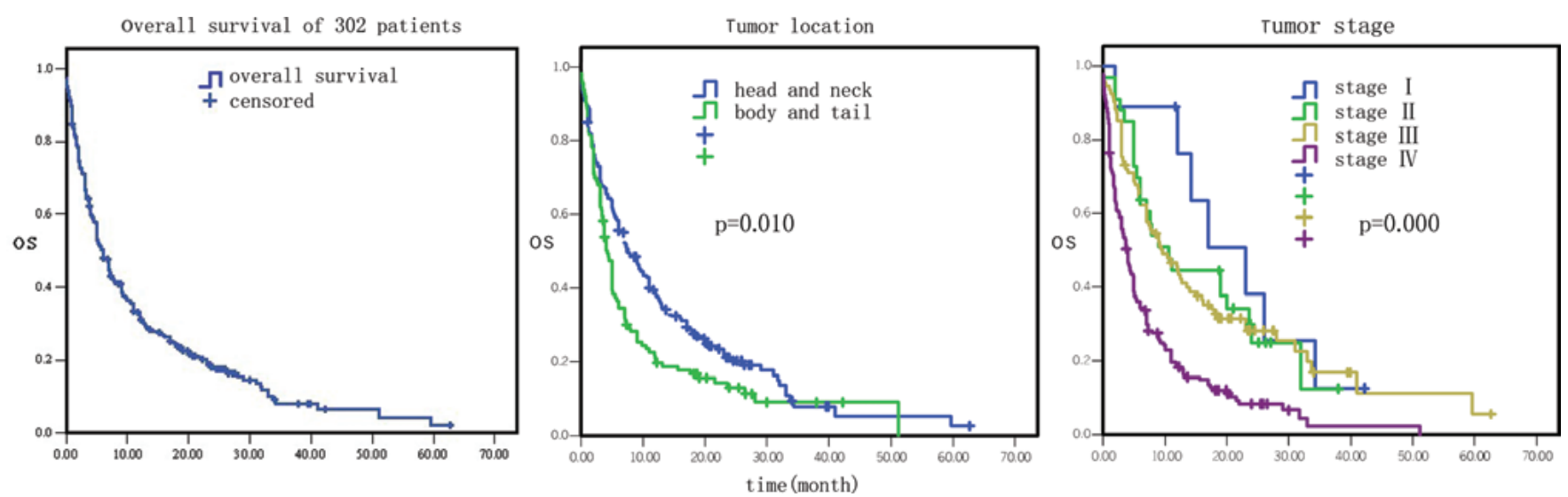

Figure 1. Overall survival and association of the primary tumor locations and tumor stage with overall survival of 302 pancreatic cancer patients analyzed using the Kaplan-Meier method.
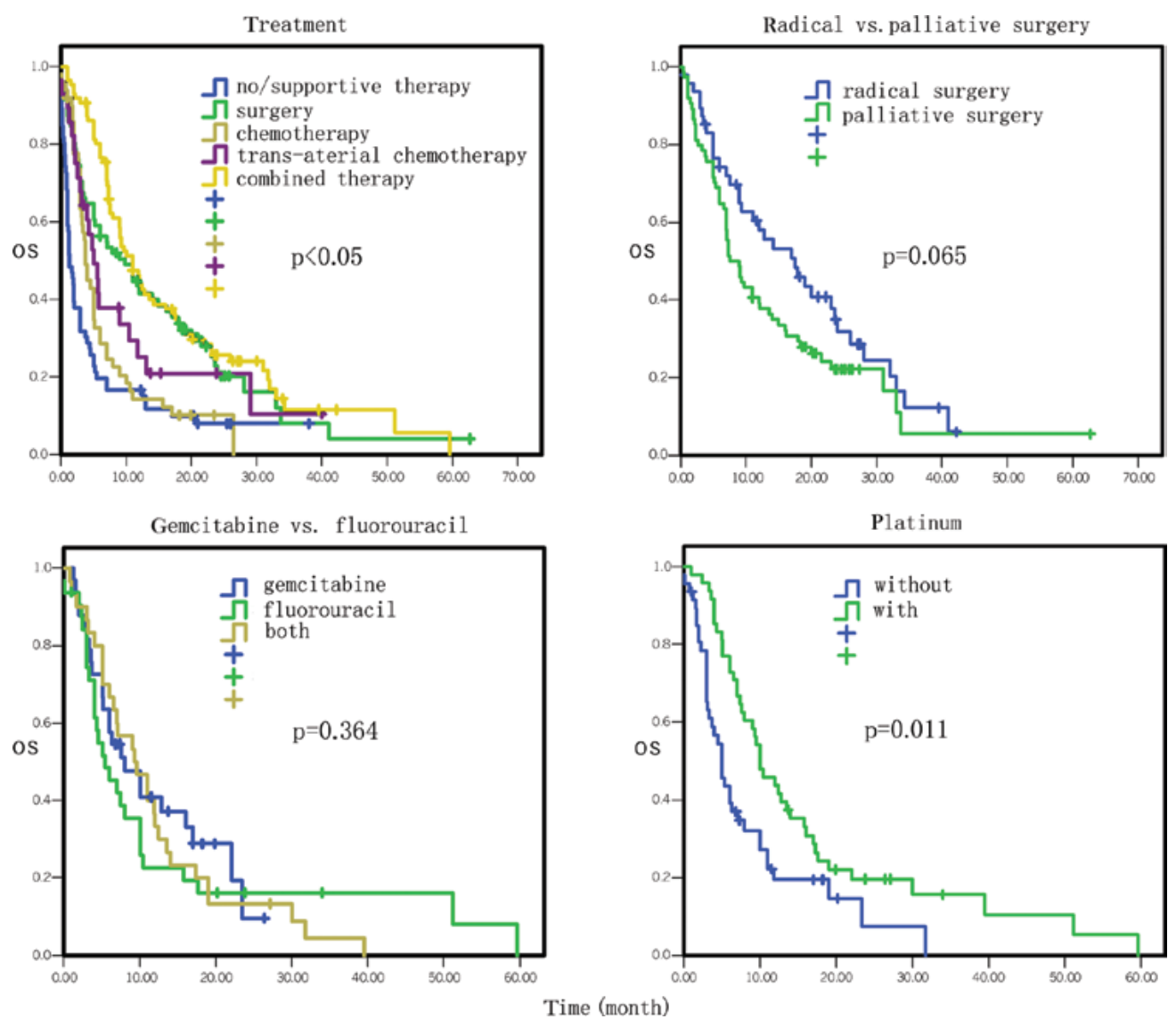

Figure 2. Comparison of the effect of treatment selections on overall survival.

The data on the association of chemotherapy with the survival of pancreatic cancer are presented in Table V. In particular, single-drug chemotherapy had no statistically significant difference in survival compared to the multi-drug regimen $(\mathrm{P}>0.05)$. Chemotherapy using gemcitabine had no statistically significant difference in survival compared to fluorouracil ( $P>0.05$; Fig. 2). However, the mortality risk of patients who received platinum chemotherapy was decreased (HR=0.56, 95\% CI 0.35-0.88, $\mathrm{P}=0.011$ ) compared to the patients who did not receive drug therapy (Fig. 2), although there was no distinction among cisplatin, carboplatin and oxaplatin $(\mathrm{P}>0.05)$.
In addition, the overall survival of pancreatic cancer patients with arterial cannula interventional chemotherapy (Table VI) was not statistically different between gemcitabine and fluorouracil $(\mathrm{P}>0.05)$.

\section{Discussion}

To date, the survival of pancreatic cancer patients remains very poor as it is usually diagnosed at the advanced stages, which are unsuitable for surgery, and chemotherapy is ineffective in the management of tumor progression and metastasis. In the present study, we investigated the factors potentially 

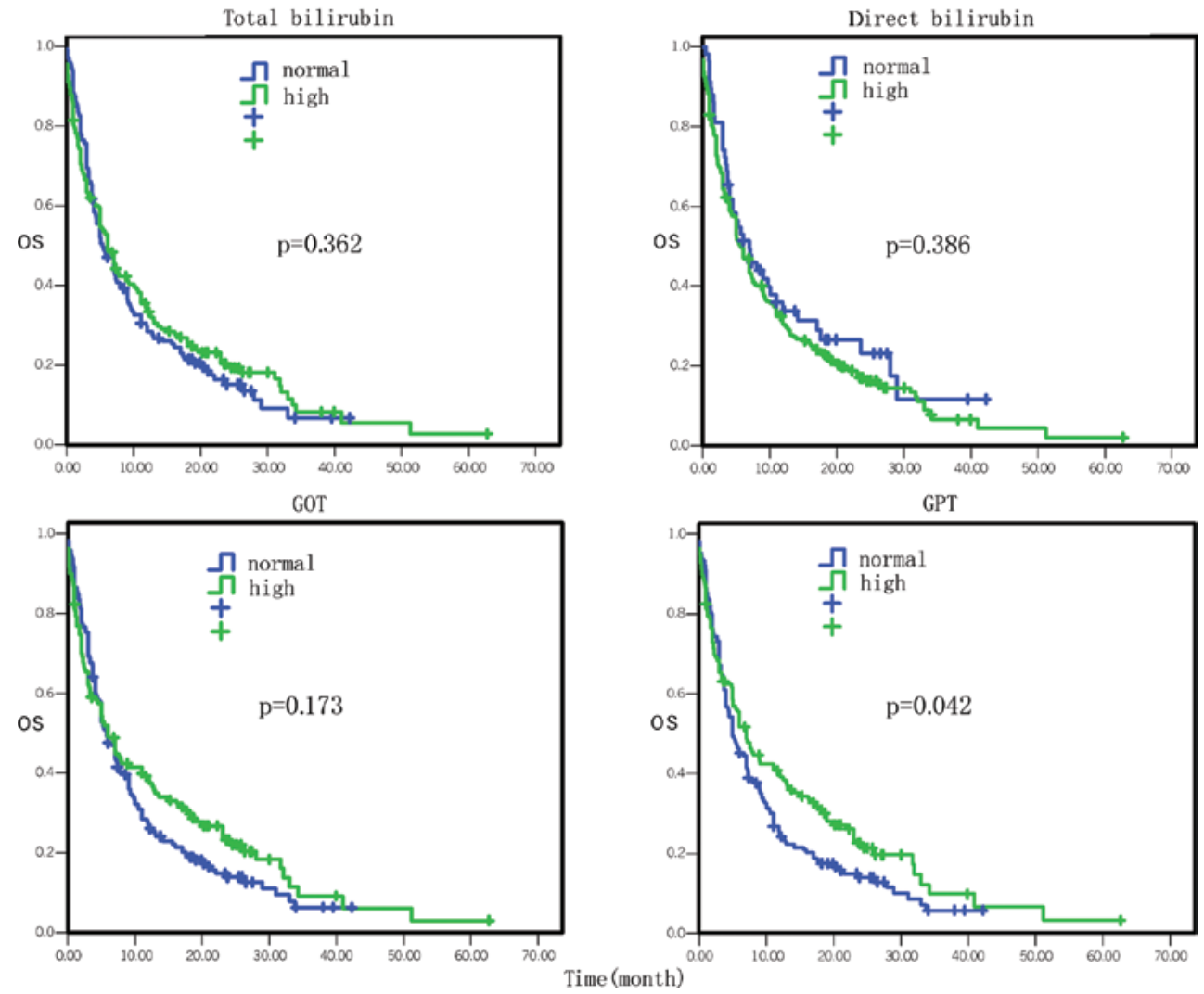

Figure 3. Comparison of the effect of liver functions on overall survival.
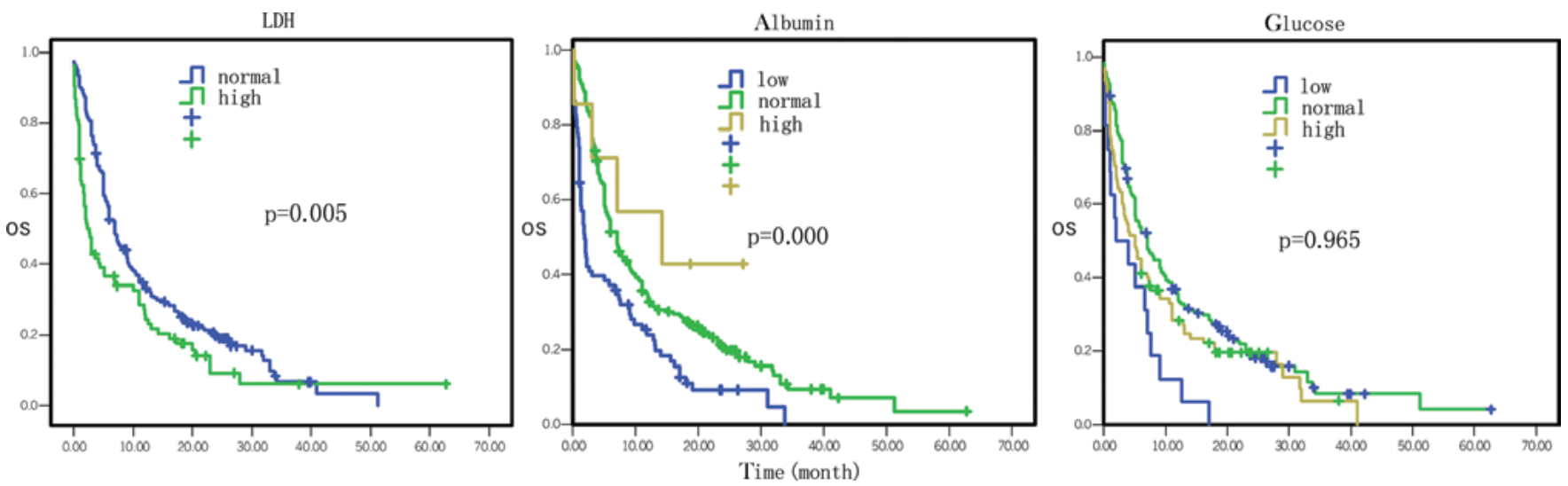

Figure 4. Comparison of the effects of lactate dehydrogenase, albumin and glucose levels on overall survival.
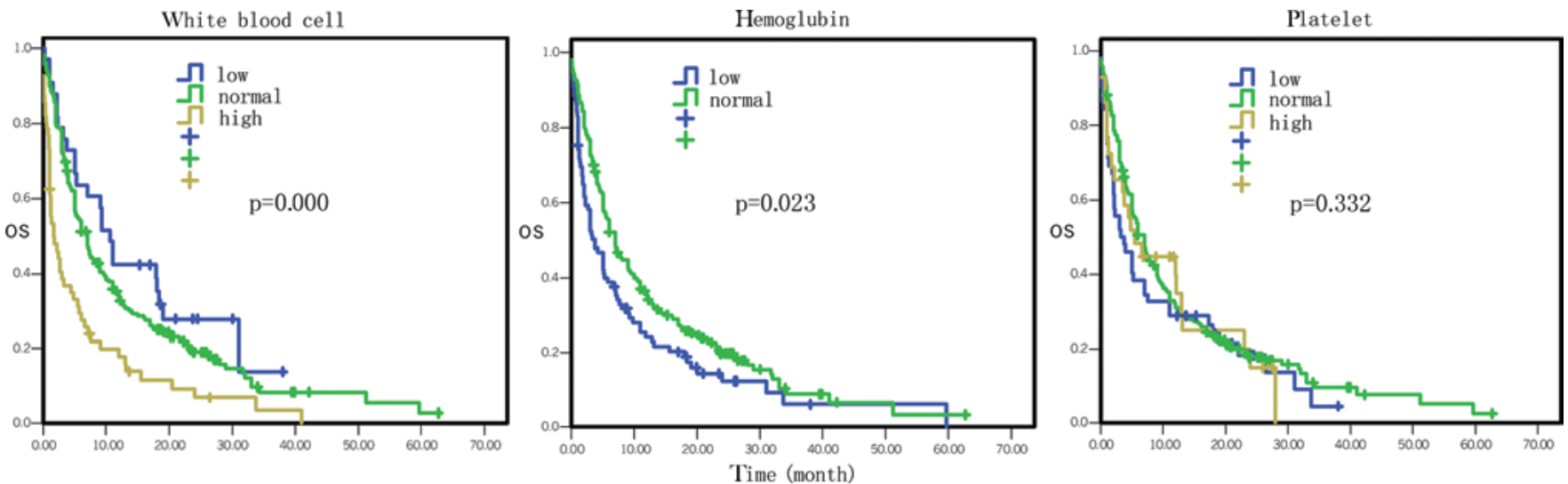

Figure 5. Comparison of the effects of white blood cell count and hemoglobin levels and platelet count on overall survival. 


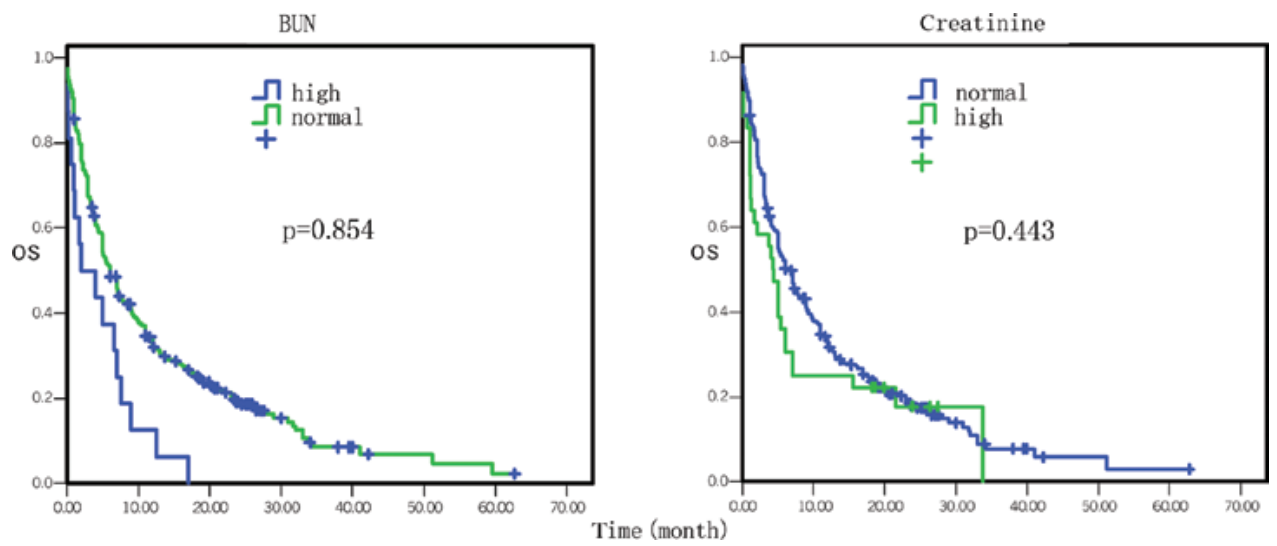

Figure 6. Comparison of the effect of kidney functions on overall survival.
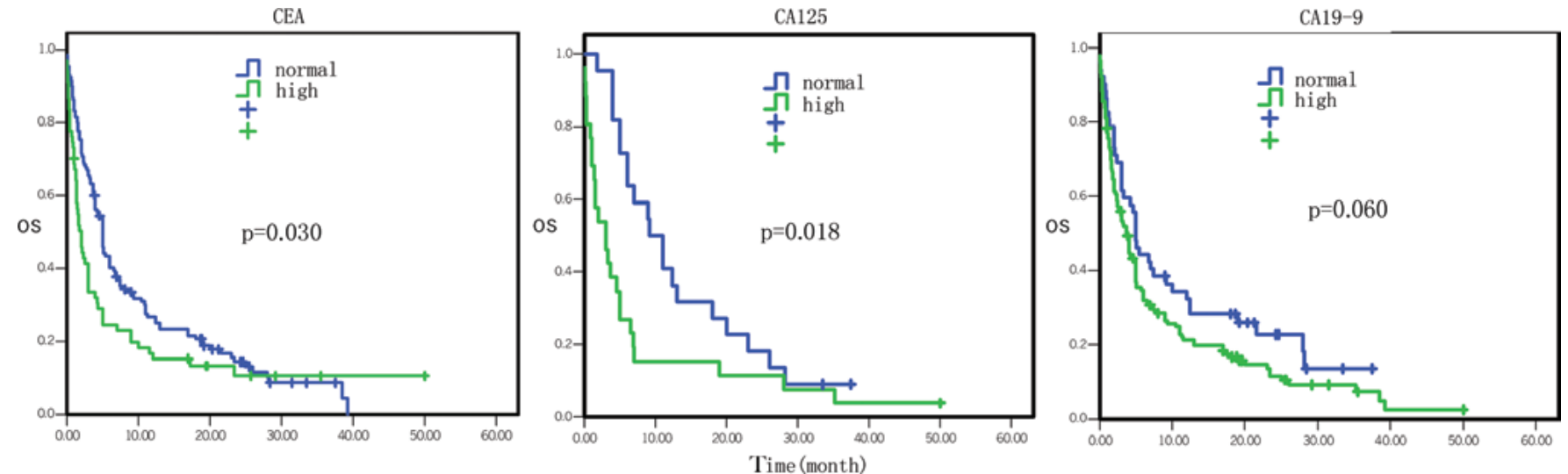

Figure 7. Comparison of the effects of various tumor markers on overall survival.

Table II. Cox multivariate regression analysis of 302 pancreatic cancer patients.

\begin{tabular}{|c|c|c|c|c|}
\hline Parameter & $\mathrm{DF}$ & Wald Chi-square test & P-value & Hazard ratio $(95 \% \mathrm{CI})$ \\
\hline Gender & 1 & 0.03 & 0.855 & $1.03(0.75-1.41)$ \\
\hline Age & 1 & 0.42 & 0.517 & $0.91(0.69-1.21)$ \\
\hline Site of primary cancer & 1 & 2.38 & 0.123 & $1.25(0.94-1.66)$ \\
\hline Stage & 1 & 13.60 & 0.000 & $1.46(1.19-1.78)$ \\
\hline Treatment & 1 & 13.93 & 0.000 & $0.51(0.36-0.73)$ \\
\hline Total bilirubin & 1 & 0.17 & 0.678 & $0.93(0.65-1.33)$ \\
\hline Direct bilirubin & 1 & 2.45 & 0.117 & $1.38(0.92-2.06)$ \\
\hline Glutamic-pyruvic transaminase & 1 & 0.05 & 0.825 & $0.94(0.56-1.58)$ \\
\hline Glutamic-oxalacetic transaminase & 1 & 0.00 & 0.970 & $0.99(0.60-1.63)$ \\
\hline Albumin & 1 & 6.10 & 0.014 & $0.62(0.42-0.91)$ \\
\hline Blood urea nitrogen & 1 & 9.81 & 0.002 & $0.43(0.25-0.73)$ \\
\hline Serum creatinine & 1 & 0.60 & 0.437 & $0.83(0.53-1.32)$ \\
\hline Lactate dehydrogenase & 1 & 2.57 & 0.109 & $1.31(0.94-1.82)$ \\
\hline Blood glucose & 1 & 3.59 & 0.058 & $0.57(0.31-1.02)$ \\
\hline Hemoglobin & 1 & 1.10 & 0.295 & $0.82(0.56-1.20)$ \\
\hline White blood cells & 1 & 6.92 & 0.009 & $1.49(1.11-2.01)$ \\
\hline Platelets & 1 & 3.94 & 0.047 & $0.73(0.53-1.00)$ \\
\hline CEA & 1 & 0.32 & 0.573 & $1.11(0.77-1.59)$ \\
\hline CA19-9 & 1 & 4.54 & 0.033 & $1.53(1.04-2.25)$ \\
\hline CA125 & 1 & 1.88 & 0.171 & $1.86(0.77-4.50)$ \\
\hline CA15-3 & 1 & 0.32 & 0.574 & $1.22(0.61-2.41)$ \\
\hline CA72-4 & 1 & 0.70 & 0.404 & $0.68(0.27-1.69)$ \\
\hline CA50 & 1 & 0.01 & 0.924 & $0.96(0.45-2.05)$ \\
\hline CA242 & 1 & 0.81 & 0.370 & $0.63(0.24-1.72)$ \\
\hline
\end{tabular}


Table III. Treatment selections and survival of pancreatic cancer patients.

\begin{tabular}{lrcrr}
\hline Parameter & No. & Median survival (months; 95\% CI) & P-value & Hazard ratio (95\% CI) \\
\hline Supportive or no therapy & 67 & $1.3(1.0-3.0)$ & & \\
Surgery & 140 & $11.0(7.8-13.5)$ & 0.000 & $0.39(0.28-0.55)$ \\
Chemotherapy & 115 & $7.3(6.0-9.3)$ & 0.000 & $0.48(0.35-0.68)$ \\
Endoscopic retrograde biliary drainage & 17 & $3.5(2.2-8.8)$ & 0.031 & $0.48(0.24-0.94)$ \\
Transcatheter arterial infusion chemotherapy & 57 & $9.0(7.3-12.8)$ & 0.000 & $0.43(0.29-0.63)$ \\
Combined therapy & 86 & $11.0(9.0-14.2)$ & 0.000 & $0.38(0.26-0.54)$
\end{tabular}

Table IV. Effect of surgical modalities on the survival of pancreatic cancer patients.

\begin{tabular}{lcccr}
\hline Parameter & No. & Median survival (months; 95\% CI) & P-value & Hazard ratio (95\% CI) \\
\hline Radical surgery & 47 & $17.6(9.3-23.6)$ & & \\
Palliative surgery & 74 & $8.3(7.0-12.1)$ & 0.065 & $1.50(0.98-2.29)$ \\
\hline
\end{tabular}

Table V. Effect of chemotherapy regimens on the survival of pancreatic cancer patients.

\begin{tabular}{|c|c|c|c|c|}
\hline Parameter & No. & Median survival (months; 95\% CI) & P-value & Hazard ratio $(95 \% \mathrm{CI})$ \\
\hline \multicolumn{5}{|c|}{ Multi-drug vs. single-drug regimen } \\
\hline Multi-drug & 58 & $7.6(6.0-10.0)$ & & \\
\hline Single-drug & 35 & $7.6(4.2-15.6)$ & 0.743 & $0.92(0.57-1.49)$ \\
\hline \multicolumn{5}{|c|}{$\begin{array}{l}\text { Gemcitabine, fluorouracil and } \\
\text { combined regimen }\end{array}$} \\
\hline Gemcitabine & 50 & $7.6(6.0-14.2)$ & & \\
\hline Fluorouracil & 25 & $5.0(3.7-10.0)$ & & \\
\hline Combined & 18 & $9.3(7.1-11.0)$ & 0.364 & $1.09(0.90-1.32)$ \\
\hline \multicolumn{5}{|l|}{ Gemcitabine } \\
\hline Yes & 68 & $9.0(7.1-11.0)$ & & \\
\hline No & 25 & $5.0(3.7-10.0)$ & 0.956 & $0.99(0.58-1.68)$ \\
\hline \multicolumn{5}{|l|}{ Fluorouracil } \\
\hline Yes & 43 & $7.4(4.5-10.0)$ & & \\
\hline No & 50 & $7.6(6.0-14.2)$ & 0.504 & $1.17(0.74-1.84)$ \\
\hline \multicolumn{5}{|l|}{ Platinum (1) } \\
\hline With & 48 & $10.0(7.6-14.0)$ & & \\
\hline Without & 47 & $5.0(3.2-7.1)$ & 0.011 & $0.56(0.35-0.88)$ \\
\hline \multicolumn{5}{|l|}{ Platinum (2) } \\
\hline Cisplatin & 8 & $7.9(5.1-17.0)$ & & \\
\hline Carboplatin & 15 & $9.6(6.0-17.3)$ & & \\
\hline Oxaliplatin & 23 & $12.4(10.0-19.0)$ & 0.093 & $0.70(0.46-1.06)$ \\
\hline
\end{tabular}

associated with the overall survival of patients, which may in turn provide a novel strategy in increasing survival. Our data showed that the median survival of the patients was 6.1 months, with 1-, 2- and 3-year survival rates of 30.1, 10.6 and 2.6\%, respectively. The Cox analysis showed that the site of primary cancer, tumor stages, treatment selections, levels of serum GPT, albumin, BUN, LDH and hemoglobin, CEA, CA19-9 and CA125, as well as WBC counts were statistically associated with overall survival. Furthermore, the median survival of patients who underwent surgery, chemotherapy, biliary drainage therapy, arterial interventional chemotherapy and comprehensive treatment with a variety of methods was better that that of patients that only underwent supportive treatment or no treatment. The mortality risk in patients who received the platinum chemotherapy regimen was reduced compared to that of patients who did not receive such a drug. The data from the present study indicate that further study is required to confirm these data, and that the use of platinum-based 
Table VI. Effect of transcatheter arterial infusion chemotherapy on the survival of pancreatic cancer patients.

\begin{tabular}{lcccc}
\hline Parameter & No. & Median survival (months; 95\% CI) & P-value & Hazard ratio (95\% CI) \\
\hline Gemcitabine & 17 & $13.1(5.8-31.0)$ & & \\
Fluorouracil & 29 & $7.3(5.1-11.0)$ & & \\
Combined regimen & 11 & $9.3(7.6-30.0)$ & 0.672 & $0.94(0.71-1.25)$ \\
\hline
\end{tabular}

chemotherapy may improve the overall survival of patients with pancreatic cancer.

Previous studies demonstrated that the median survival of pancreatic cancer patients with post-operative adjuvant therapy was 11-23 months, and that the 5-year survival rate was approximately $20 \%$. The diagnosis rate of locally advanced disease patients without distant metastasis was $15-20 \%$, with a median overall survival of 6-10 months $(11,12)$. The management of pancreatic cancer with a variety of comprehensive combined treatments, including surgery, is the primary clinical practice used presently (13). Our current data showed that the median overall survival of these patients without any treatment or only with supportive treatment was only 1.3 months. The overall survival of the patients who underwent surgery, chemotherapy, arterial interventional chemotherapy, biliary drainage and comprehensive treatment following diagnosis was increased to varying degrees. For example, the median overall survival of pancreatic cancer patients with surgery followed by other comprehensive treatments was 11.0 months, which is compatible with previously published literature (10). Of the patients who underwent surgery, those receiving radical tumor resection tended to have longer survival rates than those who underwent palliative tumor resection, although this did not reach a statistically significant difference (HR $=1.50,95 \% \mathrm{CI}$ 0.98-2.29, $\mathrm{P}=0.065$ ). Therefore, radical resection should be recommended if the patient is capable of undergoing it.

Moreover, the present study also demonstrated that the median survival of the patients treated with chemotherapy was 7.3 months, which was longer than that of patients who did not receive treatment or only received supportive treatment. In addition, there was no statistical difference in survival between single-drug and multi-drug chemotherapy regimens, and between fluorouracil- and gemcitabine-containing regimens. However, a previous study by Joo et al (14) found that chemotherapy was an independent prognostic factor for the survival of pancreatic cancer patients. Another study by Burris et al (15) compared the effect of gemcitabine with fluorouracil regimens on locally advanced and metastatic pancreatic cancer, and their data showed that the median survival of the patients who received fluorouracil treatment was 4.41 months, while that of the patients who received gemcitabine therapy was 5.65 months, but there was no statistical difference in overall survival between these two treatments.

Another study using gemcitabine monotherapy as a control, found that the combined therapy with platinum and gemcitabine did improve the progression-free survival and overall response rate in patients, but did not improve overall survival (16). These data were similar to our current findings suggesting that chemotherapy did not alter the overall survival time of pancreatic cancer patients. However, our data demonstrated that the median overall survival of the patients who received platinum reduced the mortality risk by $44 \%$. In other words, it increased the survival rate of the patients, which confirmed the data from a previous study reported by Heinemann et al that patients with gemcitabine plus platinum treatment had longer progressionfree survival and overall survival than patients receiving gemcitabine alone (17). This benefit was even greater in a subgroup of patients with a performance status of $0^{17}$. Again, our current data indicated that there was no difference among cisplatin, carboplatin and oxaplatin. The overall survival of the patients who underwent arterial interventional chemotherapy was prominently increased compared to the patients who received no treatment or only supportive treatment, whereas patients who underwent gemcitabine- and fluorouracilcontaining treatments had no difference in overall survival, and these data were similar to the Burris et al study (18).

The prognosis of pancreatic cancer is associated with a variety of factors, such as age, occupation, disease history, tumor location, surgery method, post-operative complication and TNM stage (6). Indeed, the present study showed that the site of primary cancer, tumor stages, treatments, serum levels of GPT, albumin, LDH and hemoglobin and WBC counts were independent prognostic factors using Cox univariate analysis, while Cox multivariate analysis revealed that tumor site, stage and treatment were independent prognostic factors. The poor prognosis of pancreatic cancer located in the body and tail of the pancreas is due to the fact that these tumors cause symptoms much later than those in other locations, such as the head of the pancreas. Therefore, tumors in the body and tail of the pancreas are usually at a more advanced stage at diagnosis and commonly unresectable (19). By contrast, tumors located at the head of the pancreas cause obstructive jaundice at an early stage, which usually leads to medical attention being sought much earlier, making them more curable and thus leading to a more favorable prognosis (20).

This study further showed that there was no survival difference between patients with high and normal serum levels of TB and DB, while the patients with high serum GPT levels had favorable prognosis. The latter has not been previously reported. Moreover, the median survival of the patients who had obstructive jaundice and underwent biliary drainage treatment was 3.5 months, which was 2 months longer than that of patients who did not receive any treatment or who received best supportive treatment. The reason may be that obstructive jaundice can be easily identified as a tumor in the head of the pancreas and, thus, a biliary drainage procedure would be an effective palliative treatment for such patients.

In addition, our present data demonstrated that the patients with high serum LDH levels had poor prognosis. A previous study reported by Faruk et al (21) suggested that the serum LDH levels correlated with tumor burden and reflected tumor growth and invasion potential; thus, pancreatic cancer 
patients with elevated serum LDH levels had shorter survival. Furthermore, in our study, 7 tumor-associated antigens were studied in connection with pancreatic cancer, including CEA, CA19-9, CA125, CA15-3, CA72-4, CA50 and CA242. Among these tumor markers, patients with high serum levels of CEA, CA19-9 or CA125 had poor prognosis compared to the patients with normal serum levels of these tumor markers. These data are in agreement with data from previous studies $(22,23)$. However, in our study, low WBC counts were associated with favorable patient survival, indicating that negative immunoreactions may occur in pancreatic cancer.

In conclusion, this study investigated the association of clinicopathological parameters, treatment selections and laboratory test data with the prognosis of pancreatic cancer patients. We found that these data are useful in assessing prognosis and could guide future clinical practice in the management of pancreatic cancer patients.

\section{Acknowledgements}

This study was sponsored in part by grants from the Science and Technology Commission, Shanghai Municipality and Minghang district, Shanghai Municipal Health Bureau (Nos. 2008MW23, 2009MW17 and 2010MHZ042).

\section{References}

1. Goldstein D, Carroll S, Apte M, et al: Modern management of pancreatic carcinoma. Intern Med J 34: 475-481, 2004.

2. Hirata K, Sato T, Mukaiya M, et al: Results of 1001 resections for invasive ductal adenocarcinoma of the pancreas. Arch Surg 132: 771-776, 1997.

3. Nakao A, Harada A, Nonami T, et al: Lymph node metastasis in carcinoma of the body and tail of the pancreas. Br J Surg 84: 1090-1092, 1997.

4. Shimada K, Sakamoto Y, Sano T, et al: Prognostic factors after distal pancreatectomy with extended lymphadenectomy for invasive pancreatic adenocarcinoma of the body and tail. Surgery 139: 288-295, 2006

5. Neoptolemos JP, Stocken DD, Friess H, et al: A randomized trial of chemoradiotherapy and chemotherapy after resection of pancreatic cancer. N Engl J Med 350: 1200-1210, 2004.

6. Zhang QH and Li QX: Clinical analysis of 2340 cases of pancreatic carcinoma. Natl Med J China 84: 214-218, 2004.

7. Kanda M, Fujii T, Kodera Y, et al: Nutritional predictors of postoperative outcome in pancreatic cancer. Br J Surg 98: 268-274, 2011.
8. Murakami Y, Uemura K, Sudo T, et al: Number of metastatic lymph nodes, but not lymph node ratio, is an independent prognostic factor after resection of pancreatic carcinoma. J Am Coll Surg 211: 196-204, 2010.

9. Boggi U, del Chiaro M, Croce $\mathrm{C}$, et al: Prognostic implications of tumor invasion of adhesion to peripancreatic vessels in resected pancreatic cancer. Surgery 146: 869-881, 2009.

10. Fatima J, Schnelldorfer T, Barton J, et al: Pancreatoduodenectomy for ductal adenocarcinoma: implications of positive margin on survival. Arch Surg 145: 167-172, 2010.

11. Heinemann V: Present and future treatment of pancreatic carcinoma. Semin Oncol 29: 23-31, 2002.

12. Chua YJ and Cunningham D: Adjuvant treatment for respectable pancreatic cancer. J Clin Oncol 23: 4532-4537, 2005.

13. Li D, Xie K, Wolff R, et al: Pancreatic cancer. Lancet 363: 1049-1057, 2004

14. Joo KP, Yong BY, Yong-Tae K, et al: Survival and prognostic factors of unresectable pancreatic cancer. J Clin Gastroenterol 42: 86-91, 2008.

15. Heinemann V: Gemcitabine in the treatment of advanced pancreatic carcinoma: a comparative analysis of randomized trials. Semin Oncol 29: 9-16, 2002.

16. Emilio B, Michele M, Alain G, et al: Gemcitabine-based combinations for inoperable pancreatic carcinoma: have we made real progress? A meta-analysis of 20 phase 3 trials. Cancer 110: 525-533, 2007.

17. Heinemann V, Labianca R, Hinke A, et al: Increased survival using platinum analog combined with gemcitabine as compared to single-agent gemcitabine in advanced pancreatic cancer: pooled analysis of two randomized trials, the GERCOR/GISCAD intergroup study and a German multicenter study. Ann Oncol 18: 1652-1659, 2007

18. Burris HA III, Moore MJ, Green MR, et al: Improvements in survival and clinical benefit with gemcitabine as first-line therapy for patients with advanced pancreas cancer: a randomized trial. J Clin Oncol 15: 2403-2413, 1997.

19. Takeo F, Toshio N, Naoto G, et al: Evaluation of the prognostic factors and significance of lymph node status in invasive ductal carcinoma of the body or tail of the pancreas. Pancreas 39: 48-54, 2010.

20. Ichiro W, Satoshi S, Masaru K, et al: Onset symptoms and tumor locations as prognostic factors of pancreatic carcinoma. Pancreas 28: 160-165, 2004.

21. Faruk T, Faruk A, Suleyman A, et al: Prognostic factors in pancreatic carcinoma serum LDH levels predict survival in metastatic disease. Am J Clin Oncol 24: 547-550, 2001.

22. Louhimo J, Alfthan H, Stenman UH, et al: Serum HCG beta and CA72-4 are stronger prognostic factors than CEA, CA19-9 and CA242 in pancreatic cancer. Oncology 66: 126-131, 2004.

23. Kim JE, Lee KT, Lee JK, et al: Clinical usefulness of carbohydrate antigen 19-9 as a screening test for pancreatic cancer in an asymptomatic population. J Gastroenterol Hepatol 19: 182-186, 2004. 